Horizontal collaboration in logistics: decision framework and typology

Peer-reviewed author version

MARTIN, Niels; VERDONCK, Lotte; CARIS, An \& DEPAIRE, Benoit (2018)

Horizontal collaboration in logistics: decision framework and typology. In: Operations Management Research, 11 (1-2), p. 32-50.

DOI: $10.1007 / \mathrm{s} 12063-018-0131-1$

Handle: http://hdl.handle.net/1942/25873 
This is a post-peer-review, pre-copyedit version of an article published in Operations Management Research. The final authenticated version is available online at: http://dx.doi.org/10.1007/s12063-018-0131-1

\title{
Horizontal collaboration in logistics: decision framework and typology
}

\author{
Niels Martin ${ }^{*}$, , Lotte Verdonck ${ }^{2,3}$, An Caris $^{2}$, Benoît Depaire ${ }^{1}$
}

${ }^{1}$ Research group Business Informatics, Faculty of Business Economics, Hasselt University, Agoralaan Building D, 3590 Diepenbeek, Belgium

${ }^{2}$ Research group Logistics, Faculty of Business Economics, Hasselt University, Agoralaan Building D, 3590 Diepenbeek, Belgium

${ }^{3}$ Research Foundation Flanders (FWO), Egmontstraat 5, 1000 Brussels, Belgium

\section{ABSTRACT}

Logistics service providers become increasingly aware of the need to collaborate to face challenges such as globalization and the heightened expectations of customers. This paper focuses on horizontal cooperation between logistics service providers in road transportation and introduces two novel conceptual models. Firstly, a decision framework for the development and management of horizontal logistics alliances is presented. This framework provides support to logistics service providers considering horizontal collaboration and allows for the analysis of the decision process underlying horizontal logistics alliances. The cyclical framework consists of five stages and attributes a central position to continuous evaluation. Secondly, an easy-to-use typology is proposed which defines six horizontal collaboration types based on their activity scope and degree of structural intertwinement. The typology provides logistics service providers with insights in the various horizontal collaboration types, including some key management questions. Both models are validated by knowledgeable practitioners and are applied to two real-life case studies.

\section{KEYWORDS}

horizontal cooperation; logistics service providers; decision framework; typology; case study

\section{INTRODUCTION}

Logistics service providers (LSPs) are currently faced with various challenges such as increasing competition, diminishing profit margins, complexity of new products and societal pressure to reduce the external effects of road transportation (Choi and Hwang 2015; Cruijssen et al. 2007a; Van Lier et al. 2009; Verstrepen et al. 2009). As internal efficiency and innovativeness gains tend to become scarce (Bellingkrodt and Wallenburg 2013; Cruijssen et al. 2007a), LSPs are resorting to collaboration in order to consolidate or improve their market position by sharing knowledge and other resources with partner organizations (Cruijssen et al. 2007b).

A distinction can be made between horizontal, vertical and lateral collaboration. Both vertical cooperation in supply chains and lateral cooperation in supply networks have been the focus of a multitude of research efforts over the past decades. The goal of a vertical cooperation is to establish mutually beneficial cooperations between parties operating at different levels of the supply chain offering complementary services to avoid unnecessary logistics costs (Verdonck 2017). Simatupang and Sridharan (2002) define lateral cooperation as cooperation aimed at gaining more flexibility by actively combining and sharing capabilities in both vertical and horizontal ways.

In comparison to research on vertical and lateral cooperation, the literature on horizontal cooperation in logistics remains scarce (Verdonck 2017). Horizontal logistics cooperation may be defined as cooperation between two or more firms that are active at the same level of the supply chain and perform a comparable logistics function (Cruijssen et al. 2007b). As demonstrated by a survey of Cruijssen et al. (2007a), horizontal collaboration can ameliorate the productivity of core activities, reduce supporting activity costs and allow companies to service volumes infeasible for the individual organization. Current research on the horizontal cooperation topic is primarily situated in aviation and maritime transport (Cruijssen et al. 2007b). In maritime shipping, attention is focused on conferences. These conferences are alliances of several ocean carriers that offer their services on a specific transport line against collective tariffs and identical service levels (Van Eekhout 
2002). Also in aviation horizontal cooperation constitutes a focal element, some well-known examples of major alliances are SkyTeam (20 members; SkyTeam 2017), Star Alliance (28 members; Star Alliance 2017) and OneWorld (14 members; OneWorld 2017).

Accounting for the existing research context, this paper focuses on LSPs in road transportation since horizontal cooperation provides opportunities to deal with the fundamental challenges transport organizations are currently confronted with. As horizontal collaboration between LPSs in road transportation is a fairly recent phenomenon, the research body on this topic is rather limited. Some work has been done on the operational planning of horizontal alliances between LSPs, of which an overview is presented in Verdonck et al. (2013). However, several other horizontal collaboration aspects, especially at the strategic and management level, are still scarcely researched in an LSP context. As such, this paper deals with two specific research gaps: the lack of a comprehensive decision framework for LSPs considering horizontal collaboration and the absence of a specific and easy-to-use typology outlining the main cooperation types.

To benefit from the opportunities of horizontal cooperation, an alliance needs to be established and managed. In order to perform the required formation and management activities systematically, support should be provided to LSPs considering horizontal collaboration. This is especially valuable in road transportation as LSPs tend to be small or medium-sized enterprises (Cruijssen 2007a), which can limit their access to expertise on this matter. In this respect, providing a decision framework outlining the formation and management of horizontal LSP alliances can be helpful. As stated by Ireland et al. (2002), effective alliance management poses a significant challenge which remains rather underinvestigated. With Verstrepen et al. (2009) as a notable exception, no research efforts on this topic are identified within a horizontal LSP collaboration context. This paper introduces a simple, concise, yet comprehensive decision framework which is firmly founded in literature on strategic alliance formation. It is validated using interviews with business experts and tested using two reallife case studies. Framework application and validation using expert judgment and case studies adds a dimension to the previously cited work of Verstrepen et al. (2009).

Even though horizontal alliances are developed and managed following a uniform decision framework, horizontal collaboration may be operationalized in several ways. These include, among others, joint purchasing, warehouse sharing and freight consolidation (Van Breedam et al. 2005). To add theoretical structure to the abstract horizontal collaboration concept, a typology needs to be defined. The limited research efforts on this topic by Schmoltzi and Wallenburg (2011) and Verstrepen et al. (2009) are extended here to create a pragmatic typology with six distinct collaboration types using only two dimensions. The typology allows LSPs to gain a structured insight in the wide variety of horizontal collaboration types. Moreover, it also highlights the consequences for the individual company of embarking in a particular collaboration type. Its easy-to-use structure and the specificity of the defined collaboration types distinguishes the proposed model from the identified efforts in literature. Business expert validation and case study testing is also applied for the developed typology.

In summary, horizontal collaboration between LSPs has become an important research area, since severe competition in global markets, rising costs and heightened customer expectations have caused profit margins of organizations to shrink. Engaging in a horizontal logistics cooperation provides various efficiency improving opportunities. However, collaboration projects also have significant failure rates due to their inherent complexity. It is thus essential to approach every partnership from a business perspective. In this respect, providing a decision framework outlining the formation and management of horizontal alliances and developing a classification of clear horizontal cooperation structures can be perceived as a prerequisite for collaborative success.

In this context, the contribution of this paper is threefold. Firstly, the developed decision framework provides systematic support for LSPs considering horizontal collaboration. Moreover, it allows for the analysis of the decision process underlying horizontal LSP alliances. Secondly, the novel and easy-to-use typology groups horizontal LSP alliances in a limited number of categories, enabling LSPs to gain insight in the variety of horizontal collaboration types. Finally, two real-life case studies are outlined and related to the developed conceptual models. In this way, this paper provides some necessary answers to the following relevant research 
questions "How do LSPs successfully form and manage horizontal alliances?" and "Which cooperation structures suit the needs of LSPs considering horizontal collaboration?".

Within this paper, the terms 'collaboration', 'cooperation', 'partnership', 'alliance' and 'collaborative relationship' are used interchangeably. This is in accordance with the paper of Verstrepen et al. (2009), which is most closely related to our research work.

The remainder of this paper is structured as follows. In Section 2, the applied research methodology is outlined. The third and fourth section introduce the decision framework and typology, respectively. The two case studies, used to test the developed models, are discussed in Section 5. The paper ends with a discussion and the outline of future research directions.

\section{METHODOLOGY}

This section describes the methodological underpinning of this paper. Firstly, the literature gathering approach is outlined. Secondly, the methodology supporting the performed case study research is discussed.

\subsection{Literature gathering methodology}

A thorough literature review is the starting point for the development of the decision framework and typology. In order to identify relevant literature, a two-step approach is used. Firstly, relevant literature is gathered using electronic bibliographical databases such as EBSCOHost, Web of Knowledge and Google Scholar. Regarding the decision framework, initial search terms include 'horizontal cooperation', 'horizontal collaboration' and 'horizontal alliance' combined with 'formation', 'establishment', 'process', 'management' and 'decision process'. This research phase showed that, with Verstrepen et al. (2009) as a notable exception, publications regarding the decision process in horizontal collaboration are rather scarce. Consequently, the literature scope is broadened to strategic alliances in general and search terms such as 'cooperation', 'collaboration', 'strategic alliance' and 'alliance' are added to the aforementioned list. Relevant keywords to identify literature on the horizontal collaboration typology include 'horizontal cooperation', 'horizontal collaboration' and 'horizontal alliance' on the one hand and 'typology', 'taxonomy', 'types' and 'categories' on the other hand. Secondly, the ancestry approach is applied to relevant references, i.e. the reference section of identified publications is examined. Thirdly, the descendancy approach is used to identify papers citing useful references using Google Scholar.

After gathering appropriate articles, they are critically analyzed to identify their strengths and improvement opportunities. The latter are the foundation of the proposed decision framework and typology. Moreover, literature scarcity stresses the need for the research efforts described in this paper.

\subsection{Case study methodology}

Even though case studies are increasingly used for research in the logistics field (Dinwoodie and Xu 2008), the research method is often criticized due to insufficient methodological support (da Mota Pedrosa et al. 2012). This critique is anticipated upon in this paper by formulating a research protocol ex-ante and evaluating the case study quality ex-post. The research protocol guides the case study research as recommended by, amongst others, Johnston et al. (1999), Stuart et al. (2002), Voss et al. (2002) and Yin (2009). It follows the structure proposed in Yin (2009) and outlines some general case study characteristics, fieldwork guidelines, instructions related to the interview questions and reporting issues. While a detailed discussion of the research protocol is beyond the scope of this paper, this subsection summarizes some key methodological considerations underlying the case studies. Consistent with the recommendations by Barratt et al. (2011), the remainder of this subsection is centered around the research design (Section 2.2.1), data collection (Section 2.2.2) and data analysis (Section 2.2.3). Afterwards, some more details on the case study quality evaluation are provided (Section 2.2.4). 


\subsubsection{Research design}

The developed decision framework and typology form the basis for the case study research. Within the model of Ketokivi and Choi (2014), the conducted case studies can be considered as theory testing, implying that they are used to test the literature-based decision framework and typology. More specifically, two hypotheses are tested: (i) the outlined decision framework captures the key activities required during the formation and management of a horizontal collaboration between LSPs and remains sufficiently generic such that it is applicable for LSPs operating at a different scale and (ii) existing horizontal collaborations between LSPs can be positioned within one of the categories prescribed by the typology in a transparent and straightforward way.

In order to test these hypotheses, horizontal alliances between LSPs are considered as the relevant unit of analysis. Multiple case studies need to be selected as the first hypothesis relates to the applicability of the decision framework on alliances of LSPs operating at a different scale. This is consistent with Johnston et al. (1999), stating that the selection of cases is driven by the formulated hypotheses. As an in-depth analysis of alliance formation, organization and operations is conducted, the number of case studies is limited to two (Voss et al. 2002). To select specific horizontal alliances between LSPs, a theoretical sampling approach is followed, implying that cases are selected on theoretical grounds (Eisenhardt 1989). The company size of alliance members and the geographical scope of the alliance are key selection criteria as differences with respect to these criteria might lead to diverging cooperation structures and characteristics. This will ensure the complementarity of the case studies, as recommended in Johnston et al. (1999). Taking these criteria into account, System Alliance Europe and DHB-logistiek are selected. While the former is a European alliance consisting of mediumsized LSPs, the latter operates within the Benelux (Belgium, the Netherlands and the Grand Duchy of Luxembourg) and is composed of smaller LSPs.

Regarding informant selection, a small number of informants with detailed knowledge about the alliance is targeted. This approach is supported by Voss et al. (2002), which state that a larger number of informants is required especially when the input is prone to subjectivity and respondent bias. Given the fact that mainly factual information is necessary to verify the hypotheses, a limited number of principle informants is selected. Such principle informants are characterized by having an in-depth knowledge on alliance formation, organization, operations and management. The company or alliance website and preliminary business contacts are used to support respondent identification. For the System Alliance Europe case, two informants from H. Essers, one of the founding members of System Alliance Europe, are selected: the European Network Manager and a Sales Manager. For the DHB-logistiek case study, the CEO of DHB-logistiek is selected as the principle informant. All three informants possess extensive knowledge on the alliance and have the ability to provide the required factual information on the horizontal collaboration.

\subsubsection{Data collection}

Given the hypotheses put forward for the case study research, data needs to be collected on the formation of the alliance, its structure and its management. Data will be collected by means of semi-structured interviews, supported by documents which are available on the alliance website or which can be made available by the informants.

Semi-structured interviews are used, implying that a list of key topics and questions guides the interview. However, compared to a structured interview, flexibility is present with respect to the order of questions and the potential to ask follow-up questions instigated by answers of the informants (Saunders et al. 2009). The key topics which are discussed during the interviews include the general positioning of the alliance (e.g., at a geographical level and in terms of the number of partners, partner size and the alliance scope), the motivations and experienced barriers to establish the alliance, the formation of the horizontal collaboration, the structure of the alliance, its operations and its management.

Data from documents mainly focus on maps positioning partners geographically within Europe (for System Alliance Europe) and the Benelux (for DHB-logistiek) or files containing general descriptives, e.g., related to the number of partners and the number of trucks of which the alliance can dispose. Such documents support the general positioning of the case studies. 


\subsubsection{Data analysis}

Data analysis is twofold given the two hypotheses which are investigated in the case study research. With respect to the first hypothesis, data analysis implies linking the collected data to the key steps and activities proposed in the decision framework. In this way, similarities and discrepancies between the data and the proposed framework can become apparent. Moreover, due to the theoretical sampling approach used to select the two case studies, it can be studied whether the decision framework is sufficiently generic such that it can capture both System Alliance Europe and DHB-logistiek.

Regarding the second hypothesis, data analysis involves gathering insights on the activity scope and degree of structural intertwinement of the case study. This enables the positioning of the alliance within one of the categories of the typology. The principle informants will also be asked to select a suitable alliance type. The ease with which this task is completed and the correspondence between the positioning by the researcher and principle informant conveys insights on the transparency and straightforwardness of the typology.

\subsubsection{Case study quality}

While the research protocol provides methodological support in preparation of and during the case study research, the case study quality is assessed ex-post. For this purpose, the evaluation criteria proposed by da Mota Pedrosa et al. (2012) are used: transferability, truth-value and traceability. Transferability refers to the ability to observe the case study conclusion in an alternative setting. Truth-value reflects the conformity between the input provided by interviewees on the one hand and its interpretation by the researcher on the other hand. Traceability relates to the consulted information sources and explicit documentation of the research process (da Mota Pedrosa et al. 2012). The results of the quality evaluation for the performed case study research are discussed in Section 5.3 .

\section{DECISION FRAMEWORK}

Verstrepen et al. (2009) state that, in practice, the development and management of collaborations rarely follows a structured process. To gain insight in the decision process underlying an alliance, a decision framework should be developed. This section presents related work on this matter in Section 3.1. In Section 3.2, a novel decision framework is presented.

\subsection{Related work}

As mentioned earlier, with Verstrepen et al. (2009) as a notable exception, no research efforts are identified towards modelling the decision process of horizontal collaboration between LSPs. Consequently, the literature scope is broadened towards strategic alliance formation in general.

Various publications discuss the decision process in the context of strategic alliance formation (see Table 1). However, a thorough literature analysis revealed several points for improvement in existing frameworks. Consider the model of Ring and Van de Ven (1994) as an illustration. They state that an alliance is formed by a cycle of negotiations, making commitments and the execution of these commitments. Assessment of the process steps occupies a central position within their framework. Even though the cyclical nature and the inclusion of evaluation are strengths of the model, Ring and Van de Ven (1994) do not explicitly integrate activities prior to the negotiation phase. Regarding the first steps of alliance formation, the model proposed by Whipple and Frankel (1998) can be considered. They include the awareness of the need to collaborate, its translation in generic alliance objectives and partner selection criteria, and the selection of alliance members. However, Whipple and Frankel (1998) spread partner selection activities over multiple process steps, which might lead to insufficient recognition of its importance. The framework of Bronder and Pritzl (1992) anticipates upon the observations regarding the prior models. Elements that an individual company should consider before considering collaboration, e.g. the potential benefits of the alliance, are explicitly recognized and partner selection activities are centralized in an autonomous process step. Nevertheless, Bronder and Pritzl (1992) do not explicitly recognize the implementation of agreements and the evaluation of alliance performance. Reviewing 
general operations and alliance management literature, Spekman et al. (1998) also acknowledge that there are still relevant research gaps in existing alliance formation frameworks.

The only framework that specifically deals with horizontal collaboration between LSPs is proposed by Verstrepen et al. (2009). They consider four development phases: strategic positioning, design, implementation and moderation. The first phase involves the awareness among individual LSPs to collaborate and the aspired collaboration type. In the second phase, partner selection activities are executed and the selected partners start negotiating. Next, in the third phase, a contract is drafted and the agreements are implemented. The final phase encompasses alliance management, performance evaluation and reflection on its future. The moderation phase is also connected to the design phase as e.g. an alliance expansion will require some redesign efforts. While the framework phases of Verstrepen et al. (2009) have their strengths, several weaknesses demonstrate the need for a comprehensive decision framework such as the one developed in this paper. First, Brouthers et al. (1995) state that cooperating with an unsuitable partner may be more damaging to an LSP than not collaborating at all. Based on its crucial importance, partner selection should be considered as an autonomous step in the collaborative decision framework instead of including it in a generic development phase. Second, the feedback loop considered within the framework of Verstrepen et al. (2009) (from moderation to design) could be criticized. Whenever partner companies decide to expand their cooperation, this scale increase needs to be operationalized within a new design phase. However, increasing the scale of a cooperation may also require a strategic repositioning of the collaboration, a possibility that is not acknowledged by Verstrepen et al. (2009).

The following subsection outlines a novel decision framework for horizontal collaboration between LSPs. It combines the strengths of existing models and addresses their limitations, e.g. the fact that important steps are not included or embedded in other phases. In this way, it provides a valuable tool to analyze the establishment and management of horizontal LSP alliances. Moreover, it supports LSPs considering alliance formation and e.g. leads to the identification of common alliance development bottlenecks, necessitating further research efforts.

\subsection{Decision framework overview}

This subsection presents a novel cyclical decision framework for horizontal collaboration among LSPs, consisting of five stages: orientation, partner selection, negotiation, implementation and management. Continuous evaluation occupies a central position in the framework. A visual representation of the framework is provided in Figure 1 and relevant references per stage are mentioned in Table 1. Papers marked with an asterisk focus on horizontal collaboration among LSPs. The remainder of this subsection details the tasks contained in the five framework stages, the importance of continuous evaluation and the cyclical nature.

Fig. 1 Decision framework

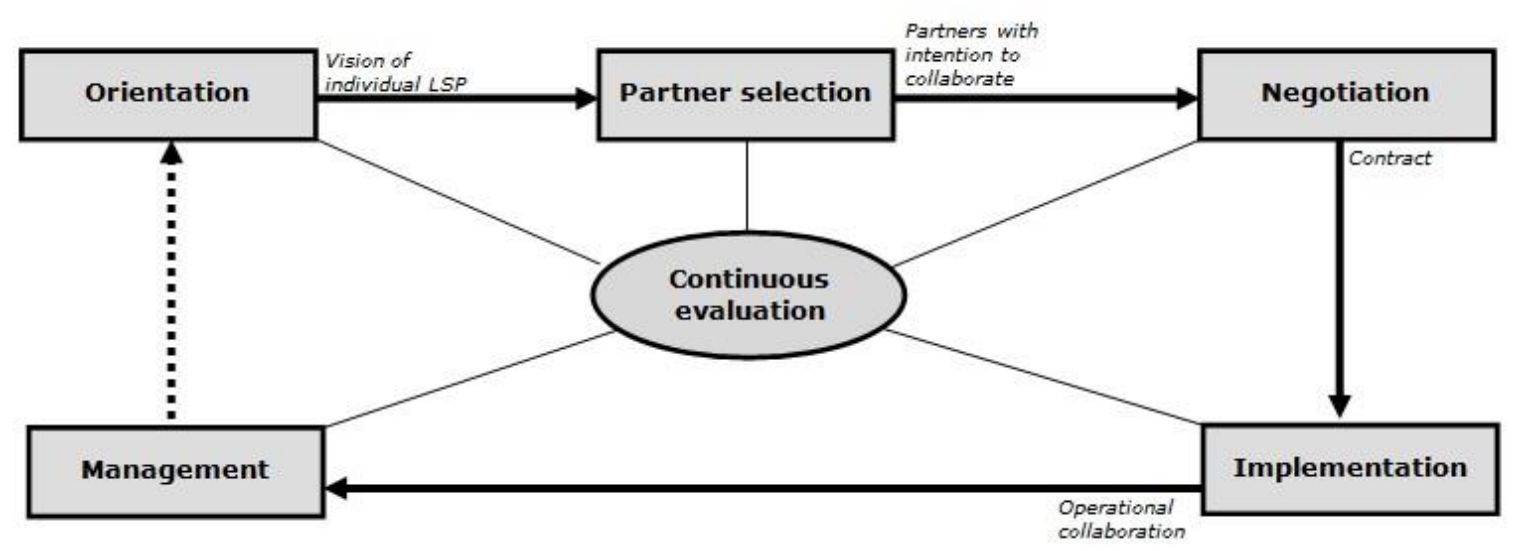


Table 1 References for each stage in the decision framework

\begin{tabular}{|c|c|c|}
\hline Stage & Reference & Article contribution \\
\hline \multirow[t]{5}{*}{ Orientation } & Bronder and Pritzl (1992) & Framework for alliance development \\
\hline & Lambert et al. (1999) & $\begin{array}{l}\text { Framework for alliance development and } \\
\text { implementation }\end{array}$ \\
\hline & Verstrepen et al. (2009)* & Typology and decision framework for horizontal \\
\hline & & LSP cooperations \\
\hline & Whipple and Frankel (1998) & Framework for alliance development \\
\hline \multirow[t]{10}{*}{ Partner selection } & Audy et al. (2012) & $\begin{array}{l}\text { Framework for development, management and } \\
\text { coordination of logistics alliances }\end{array}$ \\
\hline & Bronder and Pritzl (1992) & Framework for alliance development \\
\hline & Brouthers et al. (1995) & Framework for partner selection \\
\hline & Eisenhardt and Schoonhoven (1996) & Resource-based view on alliance formation \\
\hline & Naesens (2008) & $\begin{array}{l}\text { Decision support framework for alliance } \\
\text { implementation }\end{array}$ \\
\hline & Schmoltzi and Wallenburg $(2011)^{*}$ & $\begin{array}{l}\text { Overview of motives, structure and performance } \\
\text { attributes of horizontal LSP cooperations }\end{array}$ \\
\hline & Van Breedam et al. (2005)* & $\begin{array}{l}\text { Decision support framework for horizontal LSP } \\
\text { cooperations }\end{array}$ \\
\hline & Verstrepen et al. (2009)* & Typology and decision framework for horizontal \\
\hline & & LSP cooperations \\
\hline & Whipple and Frankel (1998) & Framework for alliance development \\
\hline \multirow[t]{4}{*}{ Negotiation } & Bronder and Pritzl (1992) & Framework for alliance development \\
\hline & Ring and Van de Ven (1994) & Framework for alliance development \\
\hline & Van Breedam et al. (2005)* & $\begin{array}{l}\text { Decision support framework for horizontal LSP } \\
\text { cooperations }\end{array}$ \\
\hline & Verstrepen et al. (2009)* & $\begin{array}{l}\text { Typology and decision framework for horizontal } \\
\text { LSP cooperations }\end{array}$ \\
\hline \multirow[t]{2}{*}{ Implementation } & Verstrepen et al. (2009)* & $\begin{array}{l}\text { Typology and decision framework for horizontal } \\
\text { LSP cooperations }\end{array}$ \\
\hline & Whipple and Frankel (1998) & Framework for alliance development \\
\hline \multirow[t]{4}{*}{ Management } & Bronder and Pritzl (1992) & Framework for alliance development \\
\hline & Schmoltzi and Wallenburg (2012)* & $\begin{array}{l}\text { Study on effects of cooperation complexity on } \\
\text { governance of horizontal LSP cooperations }\end{array}$ \\
\hline & Verstrepen et al. (2009)* & $\begin{array}{l}\text { Typology and decision framework for horizontal } \\
\text { LSP cooperations }\end{array}$ \\
\hline & Whipple and Frankel (1998) & Framework for alliance development \\
\hline \multirow{2}{*}{$\begin{array}{l}\text { Continuous } \\
\text { evaluation }\end{array}$} & Ring and Van de Ven (1994) & Framework for alliance development \\
\hline & Verstrepen et al. (2009)* & Typology and decision framework for horizontal \\
\hline
\end{tabular}




\subsubsection{Orientation}

In the rather exploratory orientation phase, an LSP has to become aware of the need to cooperate horizontally on the one hand and the opportunities that collaboration may offer on the other hand. Organizations realize that changes in their operations are required due to globalization, industry consolidation, declining profit margins or turnover reductions (Cruijssen et al. 2006; 2007a; Verstrepen et al. 2009; Whipple and Frankel 1998).

Once a company is fully convinced of the need to collaborate horizontally, a collaborative vision needs to be developed. This includes specifying the goals of the collaboration, its type and the degree of collaboration. The developed vision can be formalized in a vision text, formulated by the project team that will be responsible for the consecutive decision process phases.

To be able to judge the compatibility of potential partners in the partner selection phase, insights in the organizations' activities and characteristics is required. Hence, the LSP is advised to perform a swot-analysis identifying strengths, weaknesses, opportunities and threats (Verstrepen et al. 2009).

\subsubsection{Partner selection}

Brouthers et al. (1995) assert that insufficient time is often dedicated to the selection of an appropriate partner when strategic alliances are formed. As cooperating with an unsuitable partner is stated to be more damaging than not collaborating at all (Brouthers et al. 1995), a conscious and cautious attitude is advisable. This observation legitimizes the inclusion of partner selection as an autonomous step in the decision framework. In contrast, with Bronder and Pritzl (1992) as an exception, none of the analyzed process models insert partner selection as an independent step. These activities are included in a generic process step or spread among several stages, which tends to underestimate its importance. However, LSPs seem to be aware of the crucial importance of partner selection, as indicated in a survey by Cruijssen et al. (2007a).

Given the large number of players within the logistics sector, a preselection of LSPs is required to obtain a manageable list of potential cooperation partners. According to Naesens (2008), the criterion most often used is the compatibility in business goals.

Using the obtained candidate list as a starting point, Van Breedam et al. (2005) distinguish four key factors that should be considered during partner selection: trust and engagement, operational fit, strategic fit and cultural fit. Firstly, trust refers to each LSPs conviction that partners will refrain from opportunistic behavior. Even though trust grows over time, a minimum level should be present in this early stage of the decision process as it may influence the intensity and structure of the partners' relationship over time (Golicic and Mentzer 2011). Engagement reflects the preparedness of each partner to make a contribution to the alliance (Van Breedam et al. 2005), evoking a mutual sense of responsibility towards alliance success (Schmoltzi and Wallenburg 2012). Contributions can include financial resources, knowledge or material assets. Secondly, operational fit relates to organizational characteristics on a financial and operational level such as company size, proprietary structure and profitability. Thirdly, strategic fit implies that the organizational strategies need to be compatible, i.e. noncontradictory, and should mutually strengthen each other (Van Breedam et al. 2005). Finally, cultural fit involves compatibility between organizational cultures, which is crucial for the stability of the alliance (Audy et al. 2012; Lambert et al. 1999; Van Breedam et al. 2005). Given the intangibility of the corporate culture, cultural fit might be hard to verify. Possible indicators include the degree of customer focus, the level of environmental awareness and the management style (Van Breedam et al. 2005). In relation to this cultural fit, social aspects (i.e. contacts, reputation and the position of a firm's top management) could also influence the partner selection process. Since the top management team often serves as the initiator of the collaboration, the social position of top managers within and beyond the organization may affect its alliance formation and partner selection opportunities (Eisenhardt and Schoonhoven 1996). 
The relative importance of each of the aforementioned key factors can differ based on the LSPs cooperation objectives. Sufficient levels of trust, engagement and operational fit are the foundations of each successful collaboration. However, when an LSP wants to create a profound and enduring alliance, strategic and cultural fit gain importance. An LSP should also take into account that the degree of fit can improve or deteriorate over time, e.g. due to changes in the organizational context (Van Breedam et al. 2005).

Once potential cooperation partners are selected, all partners need to have a compatible vision on the future alliance. These congruent perspectives can be combined in a consistent global collaborative vision, which could be formalized in a written collaboration declaration.

\subsubsection{Negotiation}

Once the partners have expressed their sincere intention to collaborate, negotiations can be initiated. Some key elements included in the negotiation agenda are outlined here. An obvious focal point are the modalities of the horizontal collaboration, including the collaboration scope, required investments and operational aspects (Ring and Van de Ven 1994; Verstrepen et al. 2009). Moreover, key performance indicators (KPIs) with associated threshold values need to be defined to guide the behavior of collaborating LSPs (Simatupang and Sridharan 2005; Verstrepen et al. 2009). Ariňo (2003) distinguishes financial, operational and subjective KPIs. Another crucial negotiation topic is the division of alliance costs and benefits (Verstrepen et al. 2009). As a fair distribution of costs and benefits is an important barrier to horizontal cooperation in logistics (Cruijssen et al. 2007a), detailed arrangements are required in the negotiation phase. Literature proposes various allocation mechanisms, ranging from proportional allocations (Cruijssen et al. 2007b; Liu et al. 2010) to methods based on cooperative game theory (Cruijssen et al. 2007b; 2010; Krajewska et al. 2008; Naesens 2008).

During the negotiations, formal agreements are made on several cooperation aspects. However, horizontal collaboration cannot be founded solely on formal clauses. Interpersonal relationships, in which great importance is attributed to the alliance, are also required (Schmoltzi and Wallenburg 2012). The establishment of broad support for the alliance within the participating organizations is primordial. Due to confidentiality reasons, it might however not be possible to inform an extensive amount of staff members before a final agreement is reached (Lorange et al. 1992). During the negotiation phase, support needs to be sought from key figures within the organization. This core group might include the management of departments which will be influenced heavily by the collaboration. Prematurely gaining acceptance will, given the position of these individuals within the company, reduce the risk of alliance rejection in later decision process phases (Lorange et al. 1992).

After successful negotiations, a detailed contract outlining all collaboration modalities is drawn up. This formal document should encompass, among others, the cooperation goal, collaborative strategy and operational, financial and legal agreements. Despite the crucial importance of having a contract, drawing up a formal contract is often deemed cumbersome in practice. This can be partly attributed to the observation that collaborations often grow organically from a pilot project with a limited scope. However, the absence of a formal contract can cause problems when e.g. conflicts arise between partners. For this reason, a cooperation charter which formalizes the essence of the collaboration in generic terms can be a compromise between the advantages of a detailed contract and its associated formalities (Verstrepen et al. 2009).

\subsubsection{Implementation}

During the negotiation phase, support of key individuals should be gained. The transition towards the implementation phase enables gaining broad support in the participating LSPs. This can be done by broadly communicating the aims and opportunities of the cooperation and by transmitting a reassuring message regarding job security. The latter can avoid the perception among staff members that the alliance poses a threat (Lorange et al. 1992).

Moreover, contractual agreements are converted in practical measures in the implementation phase (Verstrepen et al. 2009; Whipple and Frankel 1998).

Implementing the collaboration needs to coincide with the set-up of adequate information and communication systems as the efficient and safe exchange of potentially sensitive data between LSPs is crucial. The degree of ICT support depends upon the scale on which partners cooperate and can necessitate significant 
investments, which might even impede alliance formation (Cruijssen et al. 2007a). Mainly for alliances with an average degree of collaboration it is doubtful if the alliance's benefits will outweigh the required investments. Conversely, expected gains probably exceed the required ICT investments when the degree of collaboration is rather high. No additional investments might be required in case of limited cooperation (Verstrepen et al. 2009).

\subsubsection{Management}

The management phase consists of activities supporting alliance functioning, which contributes to the achievement of collaborative goals and prevents opportunistic behavior. Even though some key elements will be outlined, the required management activities and style depend on the characteristics of the alliance (Schmoltzi and Wallenburg 2012).

A crucial act in the management phase is the assessment and, if necessary, the adjustment of alliance activities. To this end, the agreed upon KPIs and threshold values are used. If non-tolerable deviations from the threshold values are observed, corrective actions are required.

Besides the formal modalities, the social aspects of the alliance should also be managed. This relates, among others, to joint problem-solving and active involvement of all partners in decision-making ( $\mathrm{Li}$ et al. 2010). Moreover, conflict avoidance and conflict management are equally relevant aspects in the current framework phase. Conflicts can be averted by making clear agreements on key issues such as the protection of customer data. It is also deemed useful to organize regular meetings where all partners have the opportunity to share their worries at an early stage. Even though the term conflict has a rather negative connotation, Bronder and Pritzl (1992) state that confrontations are not destructive by definition. Discussion might create new insights which can be beneficial for the efficiency and effectiveness of the alliance activities.

\subsubsection{Continuous evaluation}

Similar to Ring and Van de Ven (1994), permanent evaluation occupies a central position in the decision framework. Alliance partners should evaluate the decision process by answering questions such as: Did we perform the swot-analysis correctly and thoroughly? Is an adequate methodology used to select a suitable partner?

A distinction can be made between individual and collective evaluation. During the orientation, partner selection and negotiation phases, only individual evaluation takes place. Every LSP verifies internally whether these process steps are executed as planned. For the implementation and management phase both individual and collective evaluation are relevant. On the one hand, each collaborating LSP has to examine to what extent their individual goals are achieved. On the other hand, alliance members have to verify collectively whether the implementation is executed smoothly, whether the cooperation is managed efficiently, etc.

\subsubsection{Cyclical nature}

Alliances typically have a dynamic character as their appearance might change over time. Consequently, multiple iterations through the outlined decision process can take place. The alliance might consider broadening the collaboration scope to benefit from additional synergies, which is consistent with the notion of organic growth mentioned by Verstrepen et al. (2009) and Whipple and Frankel (1998).

From the second iteration onwards, the scope and relative weight of decision framework steps tends to change. For instance: a collective component is added to the orientation phase after the first iteration as a collective vision on the future alliance is required. Another difference is that the collective continuous evaluation is broadened towards all framework phases in the second and subsequent iterations.

The connection between the management phase and orientation phase is represented by a dashed line in Figure 1 . This visualizes the time lag which is typically present between managing a particular collaboration and an alliance reorientation. 


\section{TYPOLOGY}

Despite the uniform decision framework, as outlined in the previous section, horizontal alliances can be operationalized in several ways. To add a theoretical foundation to the abstract concept of horizontal collaboration, a typology can be defined. Related work on this topic is briefly outlined in Section 4.1 and the novel typology is presented and discussed in Section 4.2.

\subsection{Related work}

Several authors propose a classification scheme regarding horizontal alliances in logistics. Consider e.g. Cruijssen (2006), Schmoltzi and Wallenburg (2011), Verstrepen et al. (2009) and Zinn and Parasuraman (1997). A detailed overview of all of them is omitted due to space limitations. However, Schmoltzi and Wallenburg (2011) and Verstrepen et al. (2009) are discussed as they focus on horizontal cooperation among LSPs. Verstrepen et al. (2009) define four dimensions that can be used to describe a particular horizontal LSP collaboration. Based on these dimensions, the authors describe more than ten types of horizontal collaborations. Consequently, the framework of Verstrepen et al. (2009) does not define a limited number of categories, but requires the description of a horizontal cooperation according to the four suggested dimensions. This might raise questions related to the theoretical qualification of the model as a typology. They provide a framework to describe a cooperation instead of enabling the identification of a limited number of collaboration types. Schmoltzi and Wallenburg (2011) consider six dimensions to identify six distinct collaboration types. However, the authors describe these types in very generic terms, suggesting a substantial heterogeneity within each category. Moreover, the use of six dimensions renders the structure of the typology rather complex.

In addition, research on alliance categorization within general operations management literature remains underdeveloped. Existing work on the development of alliance classifications mainly focuses on large companies, which are not representative for the LSP sector (García-Canal et al 2002; Hennart 1988; Rhoades and Lush 1997), have a mere conceptual character (Culpan 2009; Dussauge and Garrette 1997) or consider a variety of category dimensions raising questions about classification simplicity (Franco and Haase 2015).

Based on the insights gained from various publications, the following subsection outlines a comprehensive typology for horizontal collaboration among LSPs. The typology considers two dimensions and identifies six collaboration types. Our typology implicitly incorporates three (operational/tactical/strategic, competitive/non competitive, combined assets) of the dimensions described by Verstrepen et al. (2009) and four (functional, service, geographical, resource scope) dimensions introduced by Schmoltzi and Wallenburg (2011). Translating these into two key dimensions results in a clear, transparent and easy-to-use typology. Moreover, its simple structure and the specificity of the defined collaboration types distinguish the proposed typology from most of the identified efforts in literature.

\subsection{Typology overview}

The comprehensive new typology for horizontal collaborations among LSPs is visualized in Figure 2 and relevant references are mentioned in Table 2. Papers marked with an asterisk focus on horizontal collaboration among LSPs. The remainder of this subsection is organized as follows. First, the typology dimensions are discussed and, afterwards, the six resulting horizontal collaboration categories are introduced. Finally some managerial implications are discussed. 
Fig. 2 Typology

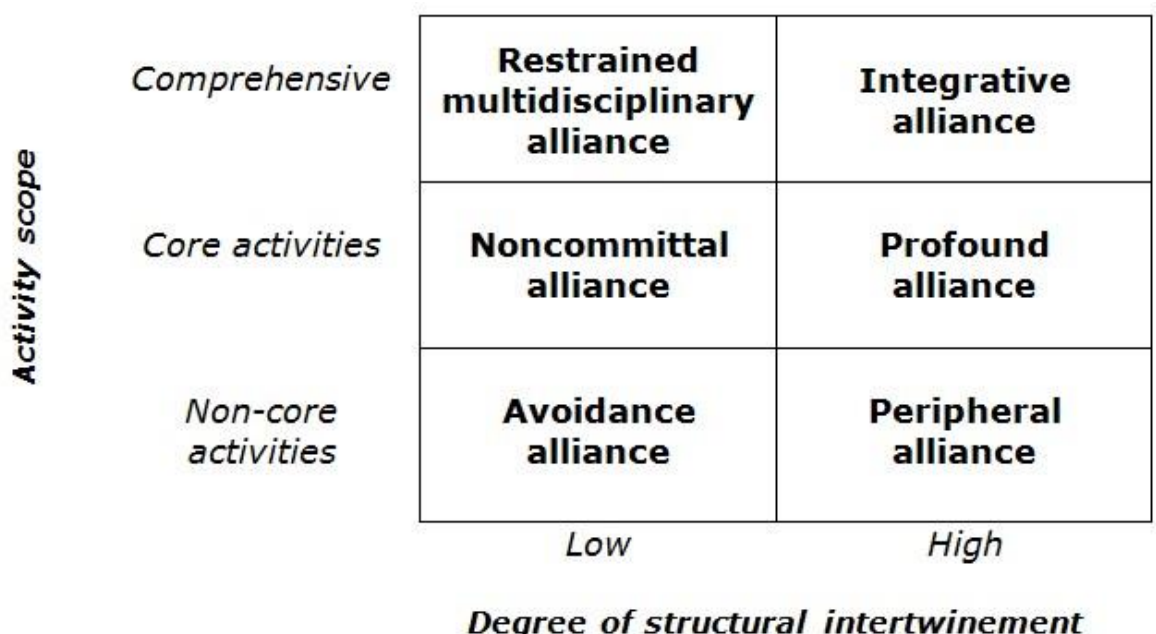

Table 2 References for classification typology

\begin{tabular}{ll}
\hline Reference & Article contribution \\
\hline Cruijssen (2006)* & Typology of horizontal LSP cooperations \\
Cruijssen et al. (2007a)* & Overview of opportunities and impediments for horizontal LSP \\
& cooperations \\
Culpan (2009) & Typology of strategic alliances \\
Dussauge and Garrette (1997) & Typology of alliances between rival firms \\
Franco and Haase (2015) & Typology of alliances between small and medium-sized enterprises \\
García-Canal et al. (2002) & Typology of global alliances \\
Hennart (1988) & Typology of equity-based alliances \\
Rhoades and Lush (1997) & Typology of strategic alliances in the airline industry \\
Schmoltzi and Wallenburg (2011)* & Overview of motives, structure and performance attributes of horizontal \\
& LSP cooperations \\
Van Breedam et al. (2005)* & Decision support framework for horizontal LSP cooperations \\
Verstrepen et al. (2009)* & Typology and decision framework for horizontal LSP cooperations \\
Zinn and Parasuraman (1997) & Typology of logistics-based alliances \\
\hline
\end{tabular}

\subsubsection{Typology dimensions}

Based on a thorough literature analysis, two key dimensions are identified to categorize horizontal collaborations among LSPs: the activity scope of the alliance and the degree of structural intertwinement among partners.

The first dimension, activity scope, refers to the domains in which LSPs join forces. The cooperation can be limited to non-core activities, e.g. purchasing tasks or truck maintenance, or can involve LSP core activities, i.e. transportation and warehousing services. A comprehensive activity scope, including both non-core and core activities, is also possible. Based on a survey among Flemish LSPs, Cruijssen et al. (2007a) state that cooperation on core activities is more appealing than a non-core activity alliance. The requirement to share sensitive order information tends to be compensated by higher expected gains (Van Breedam et al. 2005). The activity scope dimension corresponds to the functional scope in Schmoltzi and Wallenburg (2011) and the 
partnership scope in the typologies of Verstrepen et al. (2009) and Zinn and Parasuraman (1997). Cruijssen (2006) also distinguishes collaboration types depending on the level of joint activities.

The structural intertwinement among LSPs, the second typology dimension, reflects the degree to which partners' business processes are integrated, their actions are synchronized and intensive interpersonal relationships are maintained. Moreover, it determines the consequences for an LSP when the alliance's activity scope is reduced or the collaboration terminates altogether. This dimension is related to the collaboration intensity in the frameworks of Cruijssen (2006) and Zinn and Parasuraman (1997). Furthermore, the volume of joint investments and combined assets in the typology of Verstrepen et al. (2009) are included. The anticipated time frame of the alliance, as mentioned by, among others, Verstrepen et al. (2009), is implicitly embedded in the degree of structural intertwinement as a high degree of intertwinement is likely to imply a long run perspective. The typology only considers two levels of structural intertwinement, i.e. a high and a low level. Even though a less coarse specification might be useful to reflect the complex reality, priority is given to typology clarity and ease of use.

\subsubsection{Horizontal collaboration categories}

The combination of both typology dimensions leads to the identification of six horizontal collaboration categories, which are briefly discussed in the remainder of this subsection. Note that the typology does not intend to make a value judgement on the desirability of particular collaboration types. The preferable cooperation type depends upon factors such as the partners' aspirations and contextual variables including the geographical proximity of participating LSPs.

Avoidance alliance. When the activity scope of the alliance is limited to non-core activities and the degree of structural intertwinement is low, an avoidance alliance is formed. As this partnership focuses on supporting tasks, partners strive towards maximal rationalization by leveraging synergies between LSPs. This implies that the burdens related to the selected activities are spread amongst partners, e.g. at the level of investments or in terms of the required staffing. The low degree of structural intertwinement indicates that the interaction among partners, the integration of their actions and the volume of shared assets and joint investments are rather limited. The conceptual basis of the avoidance alliance is consistent with Cruijssen et al. (2007a), which state that the reduction of costs of non-core activities is a motive for horizontal cooperation. Examples of this type of cooperation are:

- Occasional joint purchases of, e.g., truck tires or office equipment such that LSPs can avoid incurring unnecessary costs due to quantity discounts.

- Running a joint recruitment agency for staff members in a foreign country to spread the costs related to getting familiar with issues such as local legislation and language.

- Regular knowledge sharing meetings during which LSPs share best practices with respect to the more efficient organization of supporting processes such as human resources and finance.

Noncommittal alliance. A noncommittal alliance involves a cooperation related to the core activities of the LSPs, but with a low degree of structural intertwinement. The noncommittal character is derived from this low degree of structural intertwinement. Since only a modest level of mutual interaction and synchronization is present, the collaboration can be ended or its composition might be altered without severe consequences for the participating LSPs. Some examples of noncommittal alliances include:

- The ad-hoc exchange of freight between LSP, e.g. due to the fact that a small load needs to be shipped to a location which is geographically far away from the customer base that a particular LSP typically serves.

- The structural exchange of freight between LSPs, but only for freight from a branch which is not of critical or strategic interest to the partners in an effort to enhance efficiency within this particular branch.

- The occasional use of a part of another LSP's warehouse, which can, e.g. be valuable to handle peak periods or to sporadically use capacity at a geographical location where an LSP is not present.

Restrained multidisciplinary alliance. When the activity scope of the alliance spans both core and noncore activities, but the degree of structural intertwinement remains low, a restrained multidisciplinary alliance is formed. The cooperation encompasses various domains but, due to the low degree of structural intertwinement, limited commitments are made which have mutual connectivity or partial integration as an ambition. A 
restrained multidisciplinary alliance can be seen as a combination of an avoidance and noncommittal alliance. Consider the following examples:

- An alliance in which freight is exchanged on an ad-hoc basis and occasional joint purchases take place.

- A collaboration in which LSPs can occasionally use each others' warehouses due to their geographical complementarity and semestrial seminars are organized to exchange best practices related to the efficiency of supporting processes.

Peripheral alliance. While the three prior collaboration types exhibit a low degree of structural intertwinement, the following three types are characterized by a high degree of structural intertwinement. This implies that partners are strongly connected at an interpersonal level and synchronize their actions. The relevant time horizon tends to be the long run due to the strong connectedness, joint investments and coordination of actions. When the activities of the alliance are limited to non-core activities, a peripheral alliance is formed. In such a cooperation, partners extensively integrate their operations for non-core activities, which reduces the required investment per partner compared to an autonomous scenario. Examples of peripheral alliances include:

- LSPs jointly investing in a centralized maintenance facility for, e.g., trucks and equipment such as forklift trucks in order to equip the facility with the required modern technologies and state-of-the-art tooling.

- A centralized administrative facility where a significant proportion of back office tasks are executed such as wage administration and invoicing.

- A highly centralized marketing policy, which is positioned at the level of the alliance and which focuses on branding and promoting the alliance instead of its individual members.

Profound alliance. An alliance which is centered around core activities with a high degree of structural intertwinement is referred to as a profound alliance. This label is selected because the alliance is centered around activities which are crucial for the continuity of the individual LSPs. The actions of partnering LSPs are highly coordinated and significant joint investments might also be performed. When the alliance would disintegrate, this would have severe consequences for the partnering LSPs given its important position within the core of their business model. Examples of this alliance type are:

- The centralization of freight planning by means of an integrated ICT-interface, based on consolidation at the alliance level.

- The centralized planning of warehouse use to enhance efficiency at the alliance level, independent of the LSP that owns the physical facilities.

- The construction and operation of joint warehouse facilities or a joint crossdock, which are used by the members of the alliance at a structural level.

- The joint development of a value-added logistics center, which provides the LSPs' customers with services such as packaging or assembly services.

Integrative alliance. An integrative alliance encompasses both core and non-core activities and has a high degree of structural intertwinement. LSPs synchronize and integrate on a broad range of domains. Given its positioning in the typology, an integrative alliance can be seen as a combination of a peripheral and a profound alliance. In this respect, examples of this cooperation type include:

- A cooperation in which a high proportion of the partners' loads are consolidated at the alliance level, a highly centralized marketing policy is established and particular administrative functions are concentrated at one particular location.

- An alliance in which LSPs jointly invest in new warehouses and high-tech maintenance facilities located in a region where partners are not yet present, while systematically using the existing warehouse and truck capacity of other partners at the alliance level.

\subsubsection{Managerial implications}

The proposed typology enables researchers and practitioners to classify alliances between LSPs and forms a foundation for future research. For LSP management, it demonstrates that horizontal collaboration can be operationalized in different ways, both with respect to the activities included and the degree of integration between partners. Moreover, the typology enables reasoning about the horizontal collaboration types and, for each alliance type, the key questions that the LSP management is confronted with. While a detailed outline on 
this matter is subject to further research, some preliminary managerial considerations are outlined in this subsection.

In an avoidance alliance, LSP management should remain aware of the option to collaborate with its partners on other non-core activities. Given the central aim to rationalize these activities, positive experiences can motivate partners to also cooperate on other non-core activities. Due to the character of these activities and the low degree of structural intertwinement, the risks for the individual LSPs of extending the collaboration are typically fairly limited. In case the avoidance alliance is operating for an extended period of time and its performance is evaluated positively, leading to trust among the partners, LSP management can consider increasing the degree of structural intertwinement by, e.g., considering joint investments in for instance a supporting system for financial administration and reporting. This would imply a move towards a peripheral alliance.

Consider, at a more general level, alliance types focusing on non-core activities, i.e. an avoidance alliance and a peripheral alliance. When sufficient cooperation experience is present and the partnership is stable, management can consider extending the alliance towards core activities. Given the strategic importance of these activities for organizational continuity and growth, this option requires careful consideration. In some alliances, it might not be opportune to take this step as it could expose an LSP's highly innovative operating procedures to the other partners. Suppose that, after an in-depth analysis, it is deemed appropriate to extend the cooperation to core activities. When this is the case, management can establish a noncommittal alliance first by, e.g. ad-hoc exchanging freight. In a second stage, mutual synchronization and shared investments can be considered, creating a profound alliance.

Managers of LSPs in alliances focusing on core activities can also examine opportunities for collaboration with respect to non-core activities. As the alliance is centered around core activities, it focuses on strategically important issues. Suppose that freight is shared within the alliance. As this requires the exchange of client information, a certain level of trust between partners is expected. Consequently, partners could consider collaborating on non-core activities as well, which can lead to, e.g. cost savings. This especially holds for alliances with a high degree of structural intertwinement as LSPs already synchronize their actions related to transportation and/or warehousing. Given the critical importance of these activities for the continuity of the organization, LSPs should investigate the savings potential of jointly executing or investing in non-core activities. This would lead to a move from a profound alliance to an integrative alliance. Within a noncommittal alliance, characterized by a low degree of structural intertwinement, it can be valuable to investigate whether efficiency gains can be reached by joining forces regarding non-core activities. This would imply that the noncommittal alliance transforms to a restrained multidisciplinary alliance.

When an LSP is currently part of a restrained multidisciplinary alliance, the activity scope encompasses both core and non-core activities. A basis of trust is likely to be present as organizations also cooperate with respect to their core activities. When the alliance is stable and evaluated consistently positive, LSP management can investigate whether it is appropriate to increase the degree of structural intertwinement between the partners. This involves, for instance, considering joint investments or a partial integration of some activities. In this respect, management can favor the option of strengthening the structural intertwinement within the alliance with respect to non-core activities first. Increasing the level of structural intertwinement would transform the restrained multidisciplinary alliance to an integrative alliance.

In an integrative alliance, management should retain a critical attitude towards the policies of the alliance. This relates, for instance, to the fact that the benefits need to be shared in a fair way. Despite the fact that formal agreements are made on this matter during alliance formation, as outlined in Section 3, changing conditions might necessitate modifications to the agreed upon mechanism to maintain its fairness. Besides gain sharing, LSP management also needs to avoid that a single partner becomes too influential within the alliance. Given the critical importance of the alliance for organizational continuity, each partner should retain sufficient influence on the strategic direction of the alliance. Even though these two issues are important for each collaboration type, it is especially relevant in an integrative alliance. 


\section{CASE STUDIES}

The validity of the decision framework and typology described above is supported by their firm foundation in existing literature. Moreover, both models have been presented to three knowledgeable practitioners. In this section, two case studies are discussed: System Alliance Europe and DHB-logistiek. Both are horizontal collaboration between LSPs with a primary focus on freight consolidation. As highlighted in Section 2.2, two hypotheses are tested: (i) the outlined decision framework captures the key activities required during the formation and management of a horizontal collaboration between LSPs and remains sufficiently generic such that it is applicable for LSPs operating at a different scale and (ii) the established horizontal collaborations between LSPs can be positioned within one of the categories prescribed by the typology in a transparent and straightforward way. At a more general level, the case studies provide an additional opportunity to validate the decision framework and typology and to exemplify its application. The use of case studies as a validation method is applied by, amongst others, Naesens et al. (2009).

Sections 5.1 and 5.2 discuss the case studies and have a similar structure. Firstly, the alliance is briefly positioned and the selected informants are retaken. Secondly, the key activities with respect to alliance formation, organization and management are reported using, whenever possible, the structure of the decision framework. Finally, the positioning of the alliance in the typology is discussed;. After the two case studies are outlined, the quality of the performed case studies are outlined in Section 5.3.

\subsection{Case study 1: System Alliance Europe}

\subsubsection{Case overview}

System Alliance Europe is a European freight consolidation network, consisting of 60 partners belonging to 24 medium-sized LSPs. Partners occupy a strong market position in their home country (System Alliance Europe 2017a; 2017b).

For this case study, interviews are conducted at $\mathrm{H}$. Essers, one of the two Belgian alliance members employing over 5400 people (H. Essers 2017). Within the organization's transportation activities, ten specialized business units can be distinguished. The activities of System Alliance Europe are situated within the four business units responsible for consolidation: consolidation Benelux, Central Europe, Southern Europe and Eastern Europe. Two respondents with relevant knowledge are interviewed at H. Essers: the European Network Manager and a Sales Manager.

\subsubsection{Discussion of the decision process}

The decision process, outlined in Section 3, is initiated by the orientation phase, which should be performed by the individual LSP. In the case of H. Essers, the need to collaborate originated from their wish to safeguard their competitive position against market players such as DHL (DHL 2017), which offer their customers unified services worldwide. Moreover, cooperation would lead to a simplification of partner relationship management as bilateral agreements and dedicated electronic data interchange connections with individual partnering LSPs became redundant. An explicit swot-analysis was not performed. Nonetheless, the outlined drivers for collaboration indicate an awareness of the organization's inability to serve the entire European market themselves, which implicitly reflects some form of swot-analysis.

Regarding partner selection, the initial alliance formation was not accompanied by extensive formal activities as bilateral agreements already existed among the 13 initial partners. Formal partner screening seemed to have limited added value since sufficient insights in, among others, operational procedures was gained by cooperating for many years. Moreover, collaboration experiences evoked a sense of trust and commitment.

The third decision framework phase, negotiations, started in 2004 among the 13 initial partners. Statutes were formulated containing the guiding principles of the alliance. Furthermore, operational and financial agreements were made, KPIs defined and selection criteria for future partners established. Regarding the alliance's management structure, a two-tier structure was established. The steering committee, composed of ten electable representatives of the partners, determines the strategic policy, while the System Alliance Europe 
Agency is responsible for policy implementation and day-to-day management. Negotiations were successfully terminated and the alliance operations started on January $1^{\text {st }}, 2005$.

The implementation phase consisted of the establishment of the required management structures and adaptations at an ICT-level to enable, among others, the transmission of confidential order data and the development of an integrated track-and-trace system. The respondents also recognized the crucial importance of gaining broad alliance support. For $\mathrm{H}$. Essers, the required efforts on this matter were mediated by the enduring bilateral contacts with alliance partners and the limited number of participating LSPs in the immediate vicinity.

In the management phase, alliance activity monitoring is an essential step. Performance assessment at System Alliance Europe is based on KPIs established during negotiations. The achievements of alliance members are evaluated on a monthly basis, after which a bonus-malus system is applied: non-complying LSPs have to pay a penalty that is distributed among complying LSPs. This practice encourages partners to improve their performance.

System Alliance Europe seems to reduce continuous evaluation to performance evaluation in the management phase. Consequently, evaluation has an explicit and formal character mainly in the management phase. In other framework steps, continuous evaluation refers to a critical attitude towards thinking patterns and analysis methods.

The framework's cyclical nature is supported as System Alliance Europe systematically increased their number of members, necessitating additional decision process iterations. From the second iteration onwards, the scope of the orientation phase has been broadened by adding a collective orientation component to the individual reflection. Related to partner selection, members were initially encouraged to invite their partners to join the network to fill geographical voids. Besides its geographical location, a potential partner also had to pass the admission criteria agreed upon in the negotiation phase. Currently, the alliance is not actively searching new partners, but the steering committee still accepts and assesses candidacies. The relative importance of the negotiation phase is reduced as potential partners have to agree on the fundamental alliance characteristics. However, operational issues such as service regions still have to be discussed.

\subsubsection{Positioning in typology}

To position System Alliance Europe in the developed typology, it should be profiled based on its activity scope and degree of structural intertwinement. Regarding the activity scope, alliance members cooperate on ourcore activities as a freight consolidation network requires using partners' transportation services and warehouses. Moreover, partners are collectively represented at logistics fares and jointly purchase gasoline and truck tires. Consequently, the cooperation is situated on the level of both core and non-core activities, i.e. the activity scope is comprehensive.

The second typology dimension, the degree of structural intertwinement, requires a division in a rather low or high level of intertwinement. Even though e.g. time tables for load exchange are synchronized and annual partner meetings are organized, the degree of structural intertwinement is rather low. Several arguments support this judgment. Firstly, alliance activities are embedded in only four of the ten transport business units of $\mathrm{H}$. Essers. The company has a solid activity basis if alliance activities should be reduced as the most profitable and future-oriented business units are beyond the System Alliance Europe scope. Secondly, the partnership has a non-exclusive character as a member can cooperate with a non-partnering LSP in a country where an alliance member is located. Thirdly, individual alliance members retain complete autonomy on several key issues such as specifying tariffs charged to partners. Finally, the absence of joint investments supports the statement that the degree of structural intertwinement is rather low.

Based on the above, System Alliance Europe can be qualified as a restrained multidisciplinary alliance. A principle informant interviewed for this case study independently reached the same positioning within the typology. 


\subsection{Case study 2: DHB-logistiek}

\subsubsection{Case overview}

DHB-logistiek is a freight consolidation network in the Benelux. In 2007, four Dutch LSPs joined forces to offer nationwide distribution services in the Netherlands. Two years later, two Belgian LSPs joined the alliance such that one-day delivery services in the Benelux could be offered. To this end, the partners currently dispose of 706 trucks, 14 warehouses and 1870 employees (DHB-logistiek 2017). One respondent is interviewed: the CEO of DHB-logistiek.

\subsubsection{Discussion of the decision process}

Collaboration was instigated by an important potential customer of a participating LSP, requesting the countrywide distribution of its products in the Netherlands. The geographical positioning of the LSP prevented accepting this request and it was not deemed feasible to open subsidiaries nationwide given the large investments required. Consequently, the LSP recognized the need to cooperate, a first milestone in the orientation phase. The alliance requirements were shaped by the potential customer's request, i.e. obtaining complete coverage of the Dutch territory. As in the prior case, awareness of the need to cooperate indicates that the individual LSP acknowledges its internal weaknesses, which can be seen as some form of implicit swot-analysis.

During initial alliance formation, limited attention was given to formal partner selection. In contrast to the previous case study, the four founding LSPs did not cooperate prior to alliance formation. A common supplier of ICT-solutions established the initial contact and subsequent personal contacts created sufficient confidence to start negotiations.

Once the aspired alliance partners were selected, negotiations were initiated. Besides a general contract, a service level agreement was developed. This one-page document included, among others, KPIs. The limited reporting style was consistent with the operational focus of the participating LSPs. Their strengths were mainly situated in the efficient execution of transport orders. Consequently, strategic considerations were often neglected. Hence, the decision was made to hand over alliance control and strategic policy definition to an autonomous management. Other points on the negotiation agenda related to partner selection criteria and service region definition for individual LSPs.

The implementation phase encompassed the operationalization of agreements and the development of broad support for the alliance. The latter automatically followed from the fundamental importance of the alliance as alliance disintegration might threaten the continuity of the individual LSPs.

In the management phase, DHB-logistiek evaluates alliance performance using the KPIs defined in the service level agreement. In contrast to System Alliance Europe, DHB-logistiek refrains from using a bonusmalus system as the interdependence between a limited number of partners results in a strong intrinsic motivation to improve performance. When the predefined goals are not met, a partner is assisted by a DHBlogistiek quality team.

As in the previous case, continuous evaluation only has a formal character when alliance performance is assessed in the management phase. In other process steps, permanent evaluation has an intangible character, rendering it difficult to observe.

The DHB-logistiek case study supports the cyclical nature of the decision framework. More specifically, the founding members reiterated through the framework when the aspired geographical scope was broadened from the Netherlands to the Benelux. As in the previous case, differences were observable between iterations. Compared to the first iteration, the orientation phase was complemented with an orientation on the alliance level. Within the context of partner selection, a list of potential partners was drawn up based on sector knowledge. Afterwards, list entries were assessed based on the complementarity of their client base, offered services, organizational structure and vision on the future. Furthermore, agreed upon admission criteria were verified. The relative importance of the negotiation phase was reduced from the second iteration onwards as the alliance foundations were not altered when e.g. a new partner was added. 


\subsubsection{Positioning in typology}

In order to position DHB-logistiek in the proposed typology, it should be categorized on both typology dimensions. The activity scope undoubtedly includes core activities as transportation services across the Benelux are jointly offered. Moreover, non-core activities such as alliance promotion and the purchase of gasoline, trucks and truck tires are performed at the alliance level. As a consequence, the activity scope is labelled as comprehensive.

To assess the degree of structural intertwinement, several arguments are taken into account. Firstly, the alliance policy is determined by the autonomous management, limiting the member's contribution to the operational execution of allocated transport orders. Secondly, LSPs heavily depend upon these orders such that alliance disintegration would threaten the organizational continuity. Thirdly, alliance members tend to identify themselves as DHB-logistiek and communication tools such as websites and business cards are standardized. Fourthly, significant joint investments are made to develop an integrated track-and-trace system and comprehensive smart phone applications. The previous discussion indicates that strong connections are present among partners. Even though day-to-day interaction between partners is limited as most communication passes through DHB-logistiek, the degree of structural intertwinement between partners is high.

By combining the positioning on both dimensions, it can be concluded that DHB-logistiek is an integrative alliance. The principle informant interviewed for this case study independently reached the same positioning.

\subsection{Case study quality}

As indicated in Section 2.2, a research protocol was developed to anticipate the criticism that case study research lacks methodological support (da Mota Pedrosa et al. 2012). In this subsection, the described cases are evaluated based on the quality criteria used by da Mota Pedrosa et al. (2012): transferability, truth-value and traceability.

First, regarding the transferability of the case study conclusion, da Mota Pedrosa et al. (2012) state that the theoretical goal, analysis unit, case selection procedure and number of considered analysis units should be clearly mentioned. This is effectuated in the developed research protocol. Related to case study selection, alliances with deviating geographical scope were deliberately chosen as their characteristics might differ. Given the presence of a research protocol, the case studies are transferable.

Secondly, the truth-value of the case studies is evaluated. Related to this criterion, the applied coding system should be outlined and justified. Coding involves using a uniform labelling system to highlight interview excerpts dealing with related matters, which facilitates the analysis of vast amounts of interview data (Layder 1998). Even though no formal coding system was developed, the research protocol categorized interview questions according to case study topics. The transcriptions followed an identical structure. Besides the coding system, discrepancies between several case studies should be indicated. Due to space limitations, the performed comparative analysis is not included, but some differences were highlighted in Section 5.2. Another truth-value element is that data gathering and analysis should alternate for validation purposes. This is taken into account by asking respondents for clarification in case knowledge gaps are present during data analysis. Finally, respondents should have the opportunity to provide feedback on the researcher's interpretation. Within this context, a draft report was provided to the respondents. It can be concluded that, even though the coding system is implicitly derived from the categorization of interview questions, the truth-value is in accordance with the criteria proposed by da Mota Pedrosa et al. (2012).

Finally, case study traceability depends to a large extent on the formulation and use of a research protocol, which is present in the current study. Furthermore, clear data collection and respondent selection rules should be defined, which are included in the research protocol. The number of respondents also has to be reported, as is done for both cases. Hence, the presented case studies are traceable.

Based on the prior discussion, it can be concluded that the presented case study research fulfils the demands underlying the quality criteria of da Mota Pedrosa et al. (2012). 


\section{DISCUSSION AND FUTURE RESEARCH}

Given the challenges LSPs are currently confronted with, they increasingly consider collaboration with fellow transport organizations in order to consolidate or strengthen their market position (Cruijssen et al. 2007a; 2007b). However, the research body on horizontal collaboration between LSPs in road transportation is limited and the available work is typically restricted to the operational level. In this respect, this paper focuses at the strategic and management level by introducing two novel models: (i) a decision framework for the development and management of horizontal alliances and (ii) a typology for those alliances.

The novel decision framework is based on an extensive literature analysis regarding strategic alliance formation. With Verstrepen et al. (2009) as an exception, no research efforts could be identified on the decision process of horizontal alliances among LSPs. However, road transportation LSPs, which tend to be small or medium-sized enterprises (Cruijssen et al. 2007a), will benefit from gaining a structured insight in the key activities that are required in alliance formation and management. The developed framework critically synthesizes literature and deals with identified points of improvement, resulting in a cyclical framework comprised of five stages: orientation, partner selection, negotiation, implementation and management. A central position is occupied by continuous evaluation. Its comprehensive nature, combined with its simple structure and concise discussion, enables the framework to provide key insights in the required activities and decisions when establishing and managing a horizontal alliance. Moreover, it adds a crucial dimension to the work of Verstrepen et al. (2009) as the presented model is applied to two real-life case studies: System Alliance Europe and DHBlogistiek.

To classify horizontal alliances between LSPs, a new typology is proposed. The typology provides a synthesized and easy-to-use categorization method by defining six collaboration types based on two dimensions: the activity scope and the degree of structural intertwinement. Besides combining the insights of existing typologies, several points for improvement resulting from existing literature are exploited. For instance: the model of Verstrepen et al. (2009) requires a description of a horizontal cooperation according to four dimensions instead of providing a limited number of categories. The proposed typology also recognizes the dynamic nature of an alliance as a partnership can belong to multiple categories over time (Verstrepen et al. 2009; Zinn and Parasuraman 1997). An alliance might move horizontally, vertically or diagonally throughout the typology. While a vertical movement indicates a broadening or narrowing of the collaboration scope, its horizontal counterpart reflects changes in the degree of structural intertwinement. The straightforward structure of the typology allows practitioners to gain insight in the various horizontal collaboration types and position their current or aspired alliance within one of the six categories. Moreover, managerial implications are presented.

Besides outlining the literature-based decision framework and typology, two case studies of horizontal cooperations between LSPs are discussed: System Alliance Europe and DHB-logistiek. The case study research is focused around two hypotheses: (i) the outlined decision framework captures the key activities required during the formation and management of a horizontal collaboration between LSPs and remains sufficiently generic such that it is applicable for LSPs operating at a different scale and (ii) the established horizontal collaborations between LSPs can be positioned within one of the categories prescribed by the typology in a transparent and straightforward way.

The case studies provide support for the first hypothesis. The key steps and activities outlined in the decision framework can be identified in both real-life alliances with their different scope, way of organization and degree of structural intertwinement between participating LSPs. The most notable deviations between the decision framework and the case studies relate to the character of the swot-analysis, partner selection and the continuous evaluation phase. Firstly, the suggested swot-analysis in the orientation phase has a more implicit character in the case studies. Secondly, regarding partner selection, no formal selection methods are used during the initial formation of the alliance. Nevertheless, the key factors raised in the decision framework on this matter are still valuable when e.g. prior cooperation experience is absent during initial alliance formation or when establishing selection criteria for future partners. Finally, with respect to continuous evaluation, the case studies seem to limit this phase to benchmarking alliance performance to the agreed upon KPIs during the management phase. This observation can be attributed to the fact that in the other phases of the decision process, evaluative 
efforts have a more implicit character, i.e. having a critical attitude towards the mindset and the analysis methods used.

The second hypothesis is also supported by the case studies. Both alliances can be positioned in the developed typology by profiling them according to the two dimensions. Insights in the typology's transparency and ease-of-use are gathered by the asking the principle informants of the case studies to select the suitable alliance type. Merely using the definitions of the dimensions, i.e. without providing additional background information and support, the principle informants suggested the same alliance type as determined by the researcher. This suggests that the typology is conceptually clear and easy to use.

Despite the sustained efforts underlying this research, some limitations need to be recognized. Firstly, the boundaries between consecutive phases in the decision framework are inherently ambiguous (Whipple and Frankel 1998). However, to structure the discussion and provide meaningful support to LSPs, a division in stages is required. Secondly, the proposed models are validated by three knowledgeable practitioners. Despite the fact the frameworks are firmly grounded in literature, consulting a broad panel of experts could enhance the support for the proposed models. However, the conscious selection of experts based on their expertise reduces the consequences of this limitation. Thirdly, the number of respondents in the case study research is two for the first case study and one for the second one. As for the previous limitation, in accordance to the research protocol, interviewees were selected based on their detailed knowledge about the alliance. Moreover, as only factual information was required, the risk of biased answers is rather limited. Finally, conducting additional case studies could make the support for the hypotheses more compelling (Johnston et al., 1999). In particular, it could enable the analysis of horizontal cooperations between LSPs situated in other cells of the typology than the cells in which System Alliance Europe and DHB-logistiek are positioned.

Based on the research results in this paper, various directions for future research can be identified. Firstly, interviews conducted to validate the decision framework showed the potential role of a neutral third party in alliance development and management. Due to a lack of trust, LSPs might be more willing to share sensitive information with a third party than with a competing LSP (Cruijssen 2012; Kampstra et al. 2006). Moreover, the third party can also guide the alliance formation process (Cruijssen 2012; Zacharia et al. 2011). To date, the position of a neutral third party has not received broad support in literature, nor in the case study research presented in this paper. According to Cruijssen (2012), the lack of literature is due to the novelty of the concept. Consequently, further research is required to determine the most appropriate phase to engage a third party and the implications of its involvement on alliance performance. Secondly, the presented typology can be extended by developing cooperation profiles for each of the six collaboration types. These profiles encompass, among others, KPIs, possible sources of conflict and potential evolution paths, which requires an extensive series of longitudinal case studies. Thirdly, further research should be performed on the relation between the degree of structural intertwinement and the merger probability of alliance members over time. It can be hypothesized that LSPs with a high degree of structural intertwinement are more likely to merge over time as their business processes become integrated and actions synchronized. Tackling this research challenge requires conducting longitudinal case studies. Finally, future work could investigate the generalizability of the proposed models. This paper focuses on horizontal collaboration between LSPs. However, it can be worthwhile to check the applicability of the introduced models to e.g. horizontal alliances in other industries or even to vertical and lateral cooperation.

\section{REFERENCES}

Ariňo A (2003) Measures of strategic alliance performance: an analysis of construct validity. J Int Bus Studies 31(1):66-79

Audy JF, Lehoux N, D’Amours S, Rönnqvist M (2012) A framework for an efficient implementation of logistics collaborations. Int T Oper Res 19(5):633-657

Barratt M, Choi T, Li M (2011) Qualitative case studies in operations management: trends, research outcomes and future research implications. J Oper Manag 29(4):329-342 
Bartolacci MR, LeBlanc LJ, Kayikci Y, Grossman TA (2012) Optimization modeling for logistics: options and implementations. J Bus Logist 33(2):118-127

Bellingkrodt S, Wallenburg CM (2013) The role of external relationships for LSP innovativeness: a contingency approach. J Bus Logist 34(3):209-221

Bronder C, Pritzl R (1992) Developing strategic alliances: a conceptual framework for successful co-operation. Eur Manag J 10(4):412-421

Brouthers KD, Brouthers LE, Wilkinson TJ (1995) Strategic alliances: choose your partners. Long Range Plann 28(3):18-25

Choi D, Hwang T (2015) The impact of green supply chain management practices on firm performance: the role of collaborative capability. Oper Manage Res 8(3-4):69-83

Coyle JJ, Langley J, Gibson BJ, Novack RA, Masodi EJ (2009) Supply chain management: a logistics perspective. South-Western, Mason

Cruijssen F (2006) Horizontal cooperation in transport and logistics. Dissertation, Tilburg University

Cruijssen F (2012) Horizontal collaboration: a CO3 position paper. CO 3 Deliverable D2.1. http://www.co3project.eu/wo3/wp-content/uploads/2011/12/CO3-D-2-1-Framework-for-collaboration-full-report-2.pdf.

Accessed 4 July 2017

Cruijssen F, Borm P, Fleuren H, Hamers H (2010) Supplier-initiated outsourcing: a methodology to exploit synergy in transportation. Eur J Oper Res 207(2):763-774

Cruijssen F, Cools M, Dullaert W (2007a) Horizontal cooperation in logistics: opportunities and impediments. Transportation Res E-Log 43(2):129-142

Cruijssen F, Dullaert W, Fleuren H (2007b) Horizontal cooperation in transport and logistics: a literature review. Transport J 46(3):22-39

Cruijssen F, Dullaert W, Joro T (2006) Logistics efficiency through horizontal cooperation: the case of Flemish road transportation companies. Vol. CentER Discussion Paper 2006-14. https://ssrn.com/abstract=895457. Accessed 4 July 2017

Culpan R (2009) A fresh look at strategic alliances: research issues and future directions. Int J Strateg Bus All $1(1): 4-23$

Da Mota Pedrosa A, Näslund D, Jasmand C (2012) Logistics case study based research: towards higher quality. Int J Phys Distr Log 4(3):275-294

DHB-logistiek (2017) DHB \& Partners: feiten en cijfers. DHB-logistiek. http://www.dhb-logistiek.com/nl/overdhb/dhb-in-cijfers. Accessed 4 July 2017

DHL (2017) Company portrait. DHL International. http://www.dhl.com/en/about_us/company_ portrait.html. Accessed 4 July 2017

Dinwoodie J, Xu J (2008) Case studies in logistics: a review and tentative taxonomy. Int J Log: Res Appl 11(5):393-408

Dussauge P, Garrette B (1997) Anticipating the evolutions and outcomes of strategic alliances between rival firms. Int Studies Manag Organ 27(4):104-126

Eisenhardt KM (1989) Building theories from case study research. Acad Manage Rev 14(4):532-550

Eisenhardt KM, Schoonhoven CB (1996) Resource-based view of strategic alliance formation: strategic and social effects in entrepreneurial firms. Organ Sci 7(2):136-150 
Franco M, Haase H (2015) Interfirm alliances: a taxonomy for SMEs. Long Range Plann 48(3):168-181

García-Canal E, Duarte CL, Criado JR, Llaneza AV (2002) Accelerating international expansion through global alliances: a typology of cooperative strategies. J World Bus 37(2):91-107

Golicic SL, Mentzer JT (2011) Transportation Relationships in the Supply Chain: Questioning Extant Theories and Methods. Transport J 50(4):315-345

Hennart JF (1988) A transaction costs theory of equity joint ventures. Strateg Manage J 9(4):361-374

H. Essers (2017) Over ons. H. Essers. http://www.essers.com/over-ons/over-h-essers/. Accessed 4 July 2017

Ireland RD, Hitt MA, Vaidyanath D (2002) Alliance management as a source of competitive advantage. J Manage 28(3):413-446

Johnston WJ, Leach MP, Liu AH (1999) Theory testing using case studies in business-to-business research. Ind Market Manag 28(3):201-213

Kampstra RP, Ashayeri J, Gottorna JL (2006) Realities of supply chain collaboration. Int J Logist Manag 17(3):312-330

Ketokivi M, Choi, T (2014) Renaissance of case research as a scientific method. J Oper Manag 32(5): 232-240

Krajewska MA, Kopfer H, Laporte G, Ropke S, Zaccour G (2008) Horizontal cooperation among freight carriers: request allocation and profit sharing. J Oper Res Soc 59(11):1483-1491

Lambert DM, Emmelhainz MA, Gardner JT (1999) Building successful logistics partnerships. J Bus Logist 20(1):165-181

Layder D (1998) Sociological practice: linking theory and social research. Sage Publications, London

Li Y, Xie E, Teo HH, Peng MW (2010) Formal control and social control in domestic and international buyersupplier relationships. J Oper Manag 28(4):333-344

Liu P, Wu Y, Xu N (2010) Allocating collaborative profit in less-than-truckload carrier alliance. J Serv Sc Ma 3(1):143-149

Lorange P, Roos J, Brønn P (1992) Building successful strategic alliances. Long Range Plann 25(6):10-17

Naesens K (2008) A swift response framework for implementing and sustaining resource pooling (applied to inventory management). Dissertation, Katholieke Universiteit Leuven

Naesens K, Gelders L, Pintelon L (2009) A swift response framework for measuring the strategic fit for a horizontal collaborative initiative. Int J Prod Econ 121(2):550-561

OneWorld (2017) OneWorld Member Airlines. https://www.oneworld.com/member-airlines/overview. Accessed 20 October 2017

Rhoades DL, Lush H (1997) A typology of strategic alliances in the airline industry: propositions for stability and duration. J Air Transp Manag 3(3):109-114

Ring PS, Van de Ven AH (1994) Developmental processes of cooperative interorganizational relationships. Acad Manage Rev 19(1):90-118

Saunders M, Lawis P, Thornhill A (2009). Research methods for business students. Pearson, Harlow

Schmoltzi C, Wallenburg CM (2011) Horizontal cooperations between logistics service providers: motives, structure, performance. Int J Phys Distr Log 41(6):552-575 
Schmoltzi C, Wallenburg CM (2012) Operational governance in horizontal cooperations of logistics service providers: performance effects and the moderating role of cooperation complexity. J Supply Chain Manag 48(2):53-74

Simatupang TM, Sridharan R (2005) An integrative framework for supply chain collaboration. Int J Logist Manag 16(2):257-274

Simatupang TM, Sridharan R (2002) The collaborative supply chain. Int J Logist Manag 13(1):15-30

SkyTeam (2017) SkyTeam Airline Alliance. https://www.skyteam.com/en/about. Accessed 20 October 2017

Spekman RE, Forbes TM, Isabella LA, MacAvoy TC (1998) Alliance management: a view from the past and a look to the future. J Manage Stud 35(6):747-772

Star Alliance (2017) Star Alliance Member Airlines. http://www.staralliance.com/en/member-airlines. Accessed 20 October 2017

Stuart I, McCutcheon D, Handfield R, McLachlin R, Samson D (2002) Effective case research in operations management: a process perspective. J Oper Manag 20(5):419-433

System Alliance Europe (2017a) Logistics with 9-star quality. System Alliance Europe. http://www.systemallianceeurope.net/en/system-alliance-europe/9-star-quality/download-brochure.html. Accessed 4 July 2017

System Alliance Europe (2017b) Partners of System Alliance Europe. System Alliance Europe. http://www.systemallianceeurope.net/en/system-alliance-europe/partners.html. Accessed 4 July 2017

Van Breedam A, Krols K, Verstrepen S (2005) Logistiek samenwerken praktisch bekeken. Flanders Institute for Logistics (VIL)

Van Eekhout B (2002) Global traffic management: beheersen van intercontinentaal transport als voorwaarde voor het realiseren van efficiënte logistieke ketens. TenHagen and Stam, Den Haag

Van Lier T, Macharis C, Caris A, Vrenken H (2009) Internal and external co-loading of outbound flows to increase the sustainability of transport: a case study. In: Witlox F, van Amstel WP (eds) Bijdragen Vervoerslogistieke Werkdagen 2009. Nautilus Academic Press, Zelzate, pp 193-208

Verdonck L (2017) Collaborative logistics from the perspective of freight transport companies. Dissertation, Hasselt University

Verdonck L, Caris A, Ramaekers K, Janssens GK (2013) Collaborative logistics from the perspective of road transportation companies. Transport Rev 33(6):700-719

Verstrepen S, Cools M, Cruijssen F, Dullaert W (2009) A dynamic framework for managing horizontal cooperation in logistics. Int J Logist Syst and Man 5(3-4):228-248

Voss C, Tsikriktsis N, Frohlich M (2002). Case research in operations management. Int J Oper Prod Man 22(2):195-219

Whipple JM, Frankel R (1998) The alliance formation process. Int Food Agribus Man 1(3):335-357

Yin RK (2009) Case study research: design and methods. Sage, Thousand Oaks

Zacharia ZG, Sanders NR, Nix NW (2011) The Emerging Role of the Third-Party Logistics Provider (3PL) as an Orchestrator. J Bus Logist 32(1):40-54

Zinn W, Parasuraman A (1997) Scope and intensity of logistics-based strategic alliances. Ind Market Manag 26(2):137-147 